\title{
MicroRNA 157-targeted SPL genes regulate floral organ size and ovule production in cotton
}

\author{
Nian Liu, Lili Tu, Lichen Wang, Haiyan Hu, Jiao Xu and Xianlong Zhang*
}

\begin{abstract}
Background: microRNAs (miRNAs) have been involved in regulation of diverse spectrum of plant development processes in many species. In cotton, few miRNAs have been well characterised in floral organ development. Floral organ, which should be finely tuned, is a crucial factor affecting the yield of cotton. Therefore, it is well worth revealing the function of miRNAs in regulation of floral organ development. Here, we report the role of miRNA156/157 in regulation of floral organ size in cotton.

Results: Over-expression of the GhmiRNA157 precursor in cotton (Gossypium hirsutum) resulted in smaller floral organs, fewer ovules and decreased seed production due to suppression of cell proliferation and cell elongation. Five SQUAMOSA promoter-binding protein-like (SPL) genes were identified as targets of GhmiRNA157 using a RNA ligase-mediated rapid amplification of CDNA end approach, and the expression level of miR157-targeted GhSPLS decreased in the miR157 over-expression lines, indicating the presence of the miR157/SPL axis in cotton. Two MADS-box genes, orthologs of AtAGL6 and SITDR8, which are associated with floral organ development and reproductive production, were repressed in the miR157 over-expression lines. In addition, auxin-inducible genes were also down-regulated, and auxin signal visualized by a DR5::GUS reporter was attenuated in the miR157 over-expression lines.

Conclusions: Our results indicate that the miR157/SPL axis controls floral organ growth and ovule production by regulating MADS-box genes and auxin signal transduction. The work further elucidates the mechanism of floral organ development and provides helpful molecular basis for improvement of cotton yield.
\end{abstract}

Keywords: Floral organ size, Gossypium hirsutum, MADS-box transcription factor, miR157, SPL gene, Ovule production

\section{Background}

Seed number is an important factor in crop yield. Because seeds are derived from fertilized ovules, development of the floral organs, especially the gynoecium, which bears ovules, directly affects the final seed number. Although environmental factors can modulate floral organ development, intrinsic mechanisms control the final size of the floral organs [1].

After the meristematic primordium is established, organ growth can be divided into two phases: cell division followed by cell expansion [2-5]. Many genes, such as the transcription factors GRFs/GIFs and JAGGED, cytochrome P450 (KLU), ubiquitin receptor $D A 1$ and

\footnotetext{
* Correspondence: xlzhang@mail.hzau.edu.cn

National Key Laboratory of Crop Genetic Improvement, Huazhong

Agricultural University, Wuhan, Hubei 430070, China

the E3 ubiquitin ligases $D A 2$ and $B B$, regulate cell proliferation rate or control the timing of proliferation arrest to maintain normal cell numbers and final organ size [6-10]. Other genes, such as the transcription factor bHLH and mediator complex subunit 8 , regulate the cell expansion rate to control the cell area, which affects organ size and shape $[11,12]$.

Moreover, hormones, such as jasmonic acid, gibberellin, brassinosteroids, and cytokinin, have been reported to be involved in the regulatory network of organ size $[3-5,13,14]$. Auxin plays a very important role in floral organ size and development. Auxin signals directly induce expression of the gene AUXIN-REGULATED GENE INVOLVED IN ORGAN SIZE (ARGOS), which further upregulates the downstream gene transcription factor AINTEGUMENTA (ANT) [15]. Over-expression of $A R G O S$ or $A N T$ can extend the period of cell division, 
resulting in larger leaves and floral organs with more seeds per silique in Arabidopsis. Conversely, mutation of $A R G O S$ or ANT decreased the final organ size and seed number [15-17]. In addition, SMALL AUXIN UP RNA $(S A U R)$ was induced by auxin to promote cell elongation and final organ size [18]. Several auxin response factors, which mediate the transcriptional response to auxin, regulate floral organ size and development. ARF8 was reported to suppress petal cell proliferation, cell elongation and final petal size through interaction with the bHLH transcription factor [19]. ARF2 could repress $A N T$ transcription to limit cell proliferation and organ size [20]. In addition to controlling floral organ size, auxin also regulates normal development of floral organs. In mutants defective in auxin biosynthesis and transport, the gynoecium is a thin and round stalk with diminished or no valve tissues of the ovary, indicating that auxin is necessary for the early establishment of carpel primordium [21]. MP (ARF5) which, mediates auxin signalling, could control ovule primordial formation by regulating $A N T$, CUC1 and CUC2 expression [22].

MADS-box genes are major players in floral organ differentiation and development. Floral homeotic proteins from MIKC-type MADS-box genes form a combinatorial quaternary complex to control differentiation of the distinct floral organs, which was used to explain the principle of the $\mathrm{ABC}(\mathrm{D}) \mathrm{E}$ model $[23,24]$. In addition to floral organ identity, MIKC-type MADS-box genes also control floral organ size and shape by regulating cell division and expansion [25]. Through chromatin immunoprecipitation (ChIP), many growth regulatory genes were shown to be targets of MADS-box transcription factors [26-28]. For example, E class MADS-box gene (SEP3) could directly bind GRF genes and JAGGED gene, which regulate cell division. In addition, MADS-box transcription factors interact with other transcription factors, such as ARF2 and SPL8 [29].

Three MADS-box transcription factor genes, APETALA1 (AP1), FRUITFULL (FUL), and SUPPRESSOR OF CONSTANS OVEREXPRESSION 1 (SOC1), were directly induced by miR156/157-targeted SPLs [30-32]. By promoting AP1, FUL and SOC1 expression, miR156/ 157-targeted SPLs could accelerate phase transition. Different miR156/157-targeted OsSPLs have been reported to regulate tiller and panicle architecture and grain size in rice [33-36]. MiR156s with highly similar miR157s are collectively referred to as miR156/157 family. Many studies have reported that miR156/157 family could regulate root development, increase tolerance to heat or salt stress, and promote trichome distribution and shoot regenerative capacity [37-40]. Here, we identified another role of miR156/157 family in the regulation of floral organ growth and ovule production through overexpression of a miR157 precursor in cotton. At least five
miR156/157-targeted SPLS and two MADS-box transcription factors, which are orthologs of AtAGL6 and SITDR8, were down-regulated in the over-expression lines. Meanwhile, auxin signalling was attenuated in the miR157 over-expression lines. We hypothesized that the miR157/SPL axis may regulate MADS-box transcription factors and affect auxin signal transduction, finally regulating floral organ growth and ovule production.

\section{Results}

Over-expression of GhmiR157 in cotton reduced flower size and seed production and altered plant architecture MiR156/157 family is one of the most conserved miRNA families in the plant kingdom and has many functions in plant development $[41,42]$. Through small RNA sequencing, the GhmiR156/157 family was profiled in cotton [43-46].

To further analyse the function of GhmiR156/157 family in cotton, we selected a GhmiR157 whose abundance is the highest in GhmiR156/157 family for analysis, and then we cloned a 372 bp genomic sequence containing a miR157 precursor from Gossypium hirsutum (Additional file 1) using the predicted reference sequence for Gossypium raimondii [46]. To overexpress GhmiR157, we used the $35 \mathrm{~S}$ promoter to drive the miR157 precursor and transferred the construct into YZ1 (Gossypium hirsutum). Seven transformants were obtained (Additional file 2), among which five showed dramatically increased mature miR157 expression levels (Fig. 1a). Compared with the nontransgenic plants (Control), the over-expression lines (OV12, 33, 35, 37, 38) showed a stronger vegetative growth vigor and produced more vegetable branches and leaves (Additional file 3). However, the size of the opened flowers and bolls from over-expression lines was notably smaller (Fig. 1b and Additional file 4B, E). In addition, the numbers of ovules per ovary were significantly decreased in the over-expression lines compared with Control, which further led to reduced seeds per boll in overexpression lines (Fig. 1c-f and Additional file $4 \mathrm{D}, \mathrm{F}$ ). Therefore, we concluded that over-expressing GhmiR157 altered plant architecture and reduced reproductive capacity sharply.

\section{Over-expression of GhmiR157 in cotton suppressed floral organ development}

Because the size of the opened flowers was significantly smaller in over-expression lines, we further compared the size of flower buds at different developmental stages among OV12, OV38 and Control (Fig. 2a). When the first-node flower on the first branch was open, we sampled first-node buds from the second branch (B2) to the tenth branch (B10) in order. Thus, buds from different branches were at different developmental stages. The results showed that at very early emergence (B10), the size 


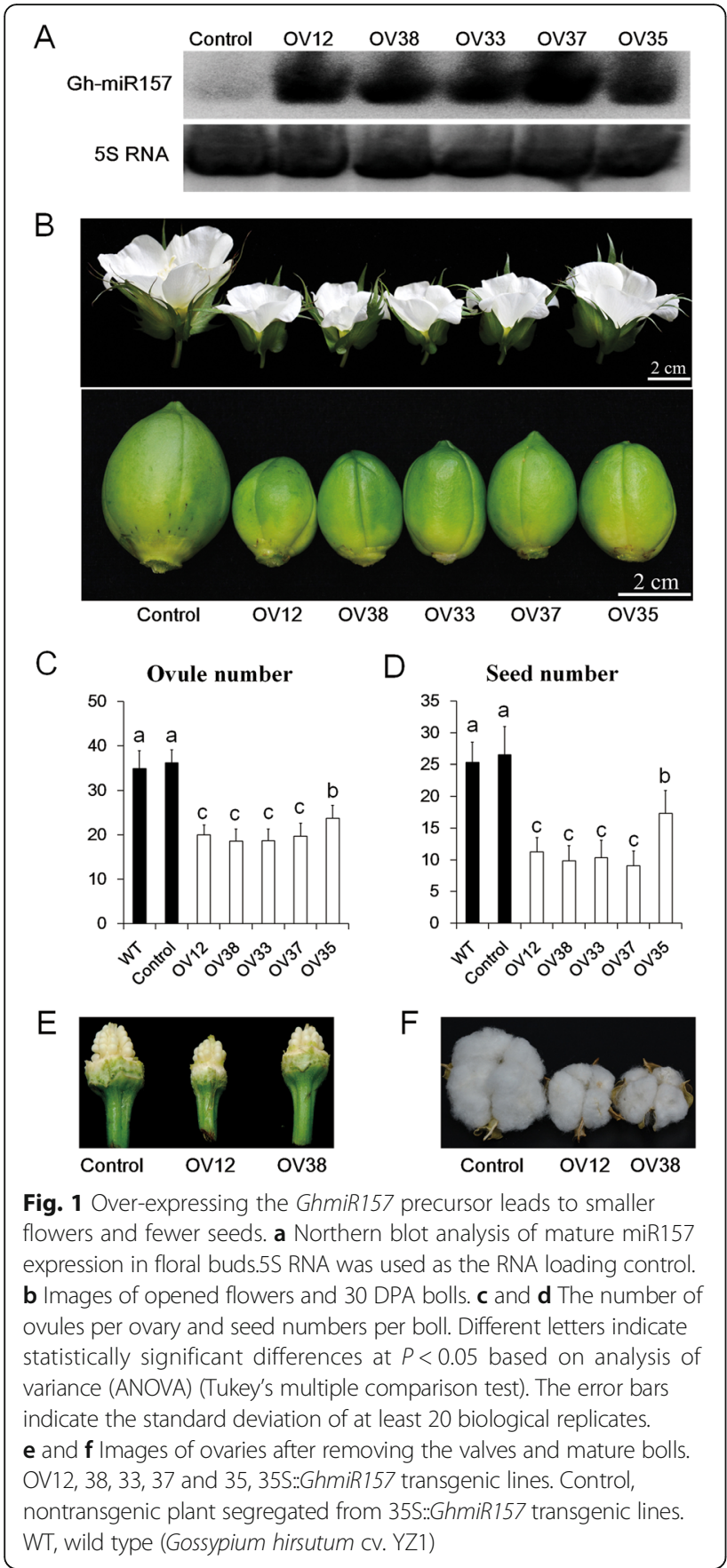

of the flower buds had become smaller in OV12 and OV38. Kinematic analysis of petal size also showed that petal development was arrested in OV12 and OV38 after flower bud emergence (Fig. 2b). In addition to petals, the other floral organs, including the bract, sepal, stigma and stamen, were also examined. The areas of the bracts, sepals and petals from opened flowers were smaller in over-expression lines (Fig. 2c, d, f, Table 1 and Additional file 4G). The anther number and the stigma length were also smaller in over-expression lines (Fig. 2e, Table 1 and
Additional file 4G). These data illustrated that the development of the four whorls of floral organs was suppressed in the over-expression lines. Because the cell number and cell size are both determinant factors of the final organ size, the epidermal cells at the adaxial side of the mature petals were examined (Fig. 2g-i). The lower cell number and smaller cell size in the over-expression lines demonstrated that cell proliferation and expansion were suppressed in over-expression lines.

\section{Ectopic expression of a GhmiR157 precursor in} Arabidopsis also arrested flower development

The miR156/157 family is highly conserved in the plant kingdom [47]. To verify whether the function of miR157 in floral organ development is universal in other species, the GhmiR157 precursor was ectopically expressed in Arabidopsis. Two successful ectopic expression lines (E4 and E6) were analysed, and the transgenic lines showed high expression of the miRNA157 precursor (Fig. 3d). Compared to the wild type, E4 and E6 had more rosette leaves (Fig. 3a), and floral organ size, especially petals, was smaller in E4 and E6 (Fig. 3b and f). Meanwhile, the shorter gynoecium contained fewer ovules in E4 and E6 (Fig. 3c and e). Thus, ectopic expression of GhmiR157 in Arabidopsis almost reproduced the phenotype in cotton, which indicated that the function of the miR156/ 157 family in floral organ development is conserved between cotton and Arabidopsis.

\section{Over-expression of GhmiR157 in cotton attenuated female fertility}

Because over-expressing GhmiR157 arrested floral organ development, we assessed whether gamete fertility was affected. Therefore, we first examined the seed set status among wild type, Control and over-expression lines in the field. The seed set efficiency of the wild type and Control was approximately $70 \%$, but over-expression lines, except OV35, had reduced efficiency, e.g., 46 to $56 \%$ (Fig. 4a), indicating that gamete fertility was defective in the over-expression lines. To determine whether the female or male fertility was defective in over-expression lines, we then used wild type pollens to pollinate Control and OV12 plants in the greenhouse. The seed set efficiency was approximately $87 \%$ in the Control $\times$ WT plants but reduced to $64 \%$ in the OV12 $\times$ WT plants, demonstrating that female fertility was reduced in the overexpression lines (Fig. 4a). Then 2,3,5-triphenyltetrazolium chloride was used to test pollen vigor, but there was no obvious difference between Control and OV12 (Fig. 4b). Thus, reduced female fertility, not male fertility, resulted in the decrease in seed set efficiency and the reduction of final seed number in over-expression lines. 

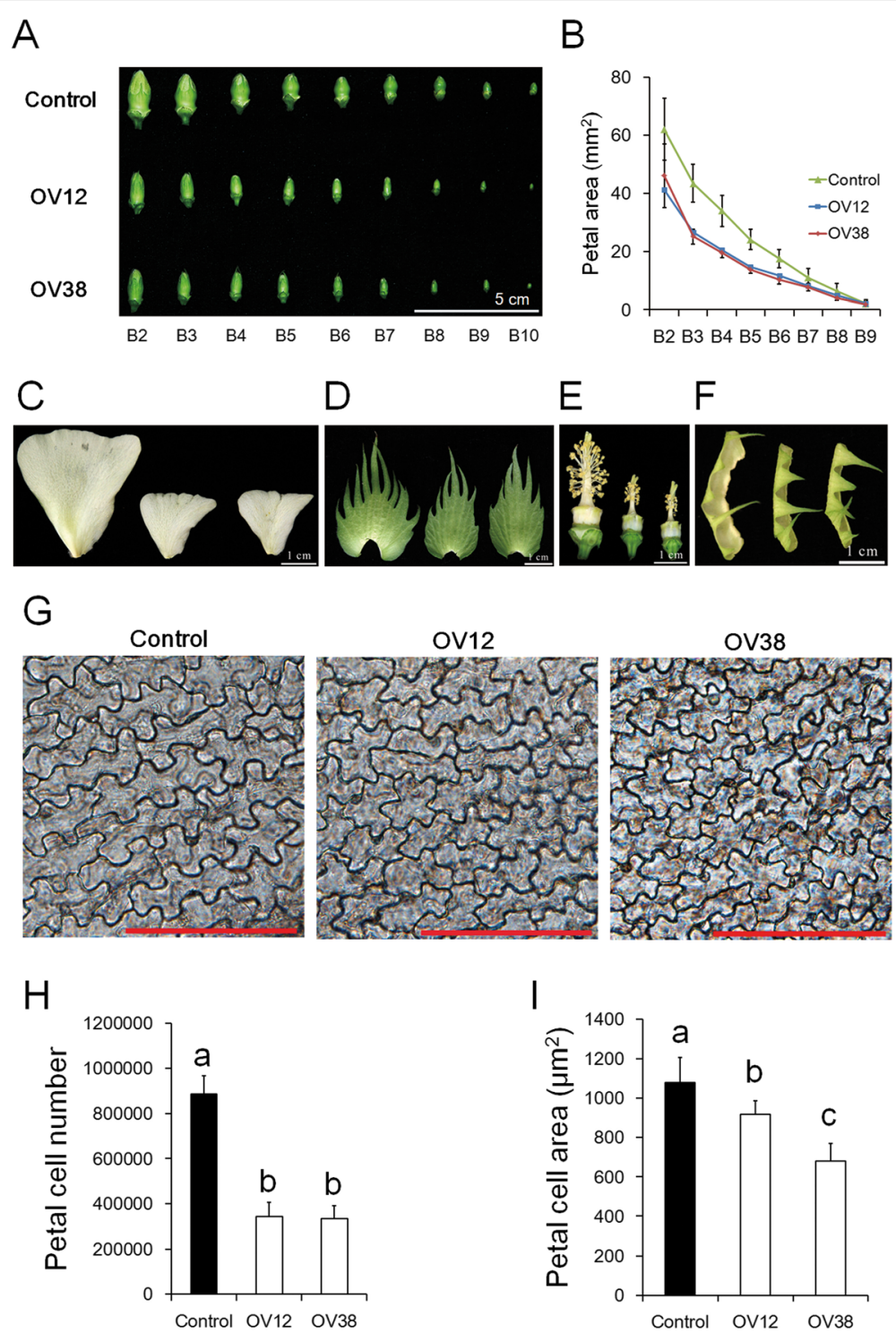

Fig. 2 Over-expressing GhmiR157 suppressed floral organ development. a Image of developing floral buds from different fruit branches. b Kinematic analysis of the developing petal area. c-f Images of the petal (c), bract (d), stamen and gynoecium (e) and sepal (f) from opened flowers of Control (left), OV12 (middle) and OV38 (right) plants. g Images of epidermal cells at the adaxial side of mature petals. Scale bar, $100 \mu \mathrm{mm}$. $\mathbf{h}$ and $\mathbf{i}$ The number of mature petal cells (h) and the mature petal cell area (i). B2-B10 represent the position of the developing floral buds from the second to tenth fruit branch. Control, nontransgenic plant segregated from 35S::GhmiR157 transgenic lines in cotton. OV12 and OV38, 35S::GhmiR157 transgenic lines in cotton. Different letters indicate statistically significant differences at $P<0.05$ based on analysis of variance (ANOVA) (Tukey's multiple comparison test). The error bars indicate the standard deviation of at least eight biological replicates

RNA-sequencing analysis of developing floral buds

To explore how over-expressing GhmiR157 suppressed floral organ development, RNA-sequencing was performed to identify differentially expressed genes between the over-expression line (OV12) and Control. Because the size of the flower buds was different between Control and OV12 at very early emergence, floral buds (length $\leq 2 \mathrm{~mm}$ ) were sampled for RNA-sequencing analysis. Approximately 12 million clean reads were generated from two biological repeat libraries of OV12 and
Control (Additional file 5). More than $85 \%$ of all the clean reads could be mapped to the cotton genome [48], and 52,005 to 52,398 genes were expressed in the libraries from the OV12 and Control plants (Additional file 5). Furthermore, 45,847 genes were expressed in all the libraries, and only 679 and 650 genes were specifically expressed in Control and OV12, respectively (Fig. 5a). The correlations of gene expression level between two biological repeats of OV12 and Control were both more than 0.97, which indicates that the biological repeats are credible (Fig. 5b). To 
Table 1 Size of floral organs in Null and miR157 over-expressing lines

\begin{tabular}{llll}
\hline & Control & OV12 & OV38 \\
\hline Petal area $\left(\mathrm{mm}^{2}\right)$ & $965.29 \pm 67.83^{\mathrm{a}}$ & $328.77 \pm 87.07^{\mathrm{b}}$ & $258.49 \pm 59.48^{\mathrm{b}}$ \\
Bract area $\left(\mathrm{mm}^{2}\right)$ & $553.71 \pm 72.36^{\mathrm{a}}$ & $339.60 \pm 110.91^{\mathrm{b}}$ & $342.29 \pm 83.92^{\mathrm{b}}$ \\
Stigma length $(\mathrm{mm})$ & $19.26 \pm 0.99^{\mathrm{a}}$ & $10.43 \pm 1.26^{\mathrm{b}}$ & $10.85 \pm 1.23^{\mathrm{b}}$ \\
Anther number & $66.56 \pm 6.68^{\mathrm{a}}$ & $17.60 \pm 3.22^{\mathrm{b}}$ & $17.64 \pm 2.87^{\mathrm{b}}$ \\
Sepal length (mm) & $27.80 \pm 1.57^{\mathrm{a}}$ & $20.87 \pm 2.42^{\mathrm{b}}$ & $19.85 \pm 1.68^{\mathrm{b}}$ \\
Sepal width (mm) & $16.87 \pm 2.75^{\mathrm{a}}$ & $12.69 \pm 1.30^{\mathrm{b}}$ & $11.77 \pm 3.11^{\mathrm{b}}$
\end{tabular}

Control is the nontransgenic plant segregated from the 35S::GhmiR157 transgenic lines in cotton. OV12 and OV38 are 35S::GhmiR157 transgenic lines in cotton. Values are shown as the mean \pm standard deviation. In each row, values with different superscipt letters are significantly different based on Tukey's multiple comparison test $(P<0.05)$

screen differentially expressed genes between Control and OV12, we used the NOISeq package [49]. Genes were filtered based on following criteria: fold change $\geq$ 2 and divergence probability $\geq 0.8$. We identified 539 differentially expressed genes, in which 368 genes were up-regulated in Control, but only 171 genes were down-regulated in control (Additional file 6). Based on another method, the EBSeq package [50], the results also showed that more up-regulated genes were found in Control than in OV12 (Fig. 5c).

In the up-regulated genes of Control, some are transcription factors, such as SPL and MADS-box genes. Interestingly, all the differentially expressed SPLS have been predicted as targets of GhmiR157, but the abundance of non-miR157-targeted SPLs was not different between Control and OV12 (Additional files 6 and 7). There are 59 members of the SPL family, which could be categorized into eight subgroups based on orthologous genes in Arabidopsis, and five of eight subgroups could be predicted as targets of GhmiR157 (Additional files 8 and 7). Five $S P L s$, which were selected from five subgroups, were further verified as GhmiR157 targets using RLM-RACE (Fig. 5n). Real-time PCR was performed to validate the abundance of these five SPLs. The results showed that compared to Control, three SPLs (Gh_A10G2217, Gh_A11G0344 and Gh_A13G0749) were significantly down-regulated in OV12 and OV38, and the expression level of other SPLs (Gh_A01G2095 and Gh_A01G1281) modestly decreased in OV12 and OV38 (Fig. 5d-h). These data demonstrated that GhmiR157-targeted SPLs were generally down-regulated in over-expression lines.

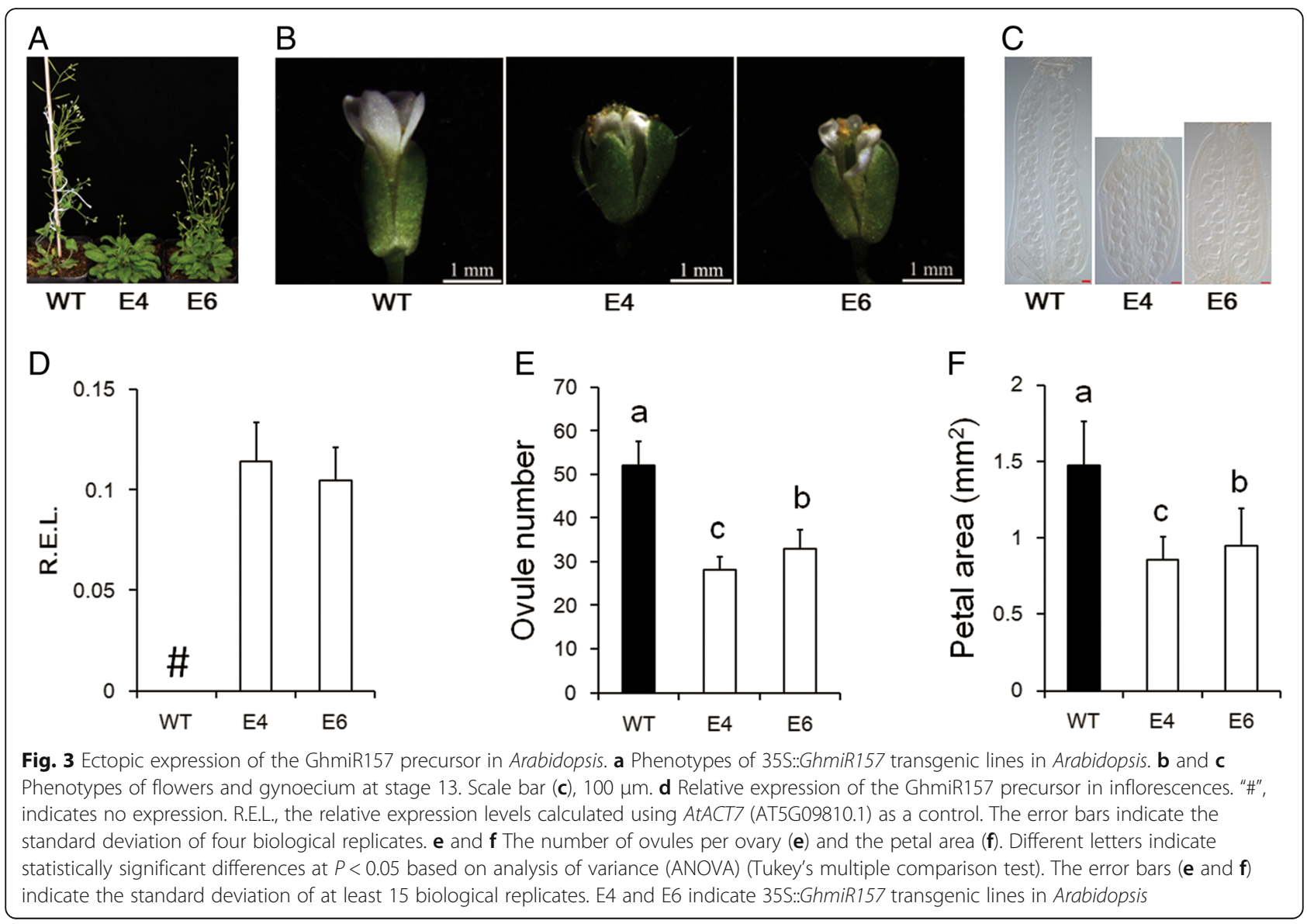



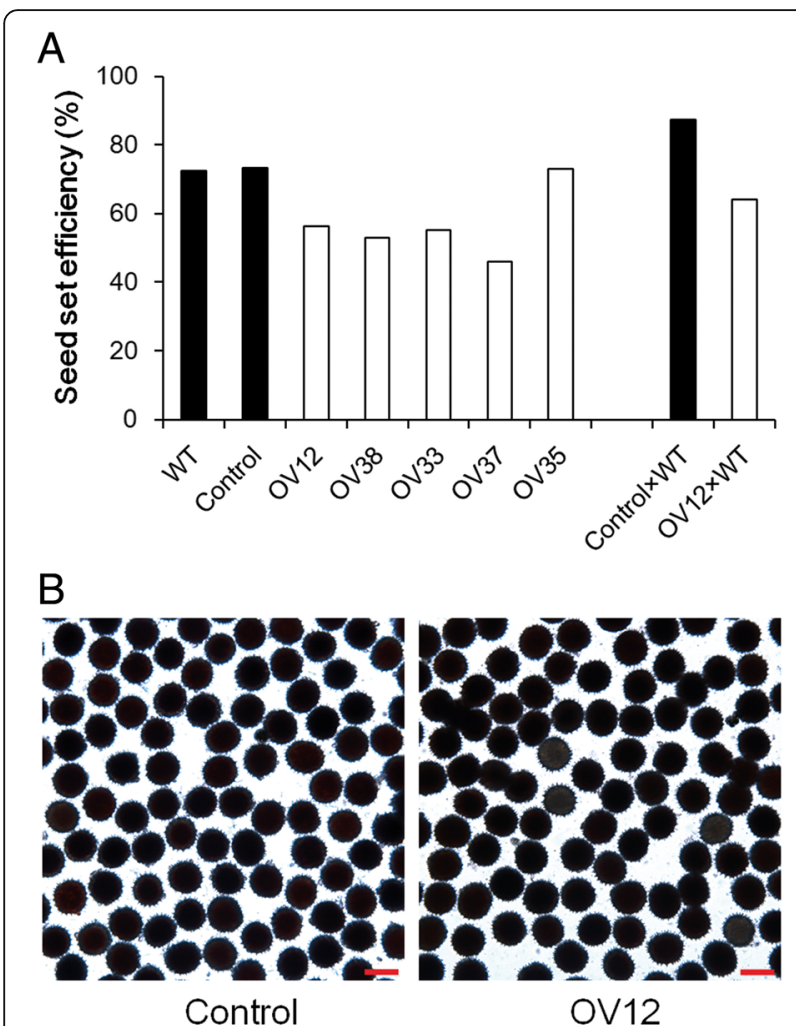

Fig. 4 Fertility test of 35S::GhmiR157 transgenic lines. a Calculation of seed set efficiency $(100 \% \times$ seed/ovule). $\mathbf{b}$ Image of pollen stained with 2,3,5-triphenyltetrazolium chloride. Control, nontransgenic plant segregated from 35S::GhmiR157 transgenic lines. OV12, 38, 33, 37 and 35, independent 355::GhmiR157 transgenic lines. WT, wild type (Gossypium hirsutum cv. YZ1). Control $\times W T$ and OV12 $\times W T$ indicate the plants from Control and OV12 were pollinated with wild type pollens in the greenhouse

MADS-box transcription factor genes, such as AtSOC1, $A t A P 1$ and $A t F U L$, were shown to be directly up-regulated by SPLs in Arabidopsis [30-32]. Our data showed that three MADS-box transcription factors, Gh_A11G0755, Gh_D08G1430 and Gh_A11G0343, which were orthologs of AtSOC1, AtAGL6 and SITDR8, respectively, were significantly down-regulated in OV12 and OV38 compared to Control (Fig. 5i-k). The levels of Gh_D13G0878 and Gh_A07G0605, which are orthologs of AtAP1 and AtFUL, were slightly lower in OV12 and OV38 than Control (Fig. 51 and m). Similarly, AtAP1, AtFUL, AtSOC1, and AtAGL6 were also down-regulated in 35S::GhmiR157 Arabidopsis transgenic lines compared with the wild type (Additional file 9). All the data indicated that MADS-box genes, as candidate downstream genes of GhmiR157-targeted SPLs, were down-regulated in over-expression lines.

\section{Expression pattern of GhmiR157 and miR157-targeted GhSPLs during floral bud development}

Floral organ development in cotton lasts approximately a month from floral bud emergence to flower opening.
Based on floral bud length, we identified seven stages of floral bud development before flower opening (Fig. 6n). Ovules appeared at the 4-6 mm floral bud stage. After that, the floral bud, including the differentiated four floral whorls, continued growing to approximately $25 \mathrm{~mm}$ in length before the flower opened. GhmiR157 expression was very low at the $0-2 \mathrm{~mm}$ and $2-4 \mathrm{~mm}$ stages, but it was expressed at much higher levels at the 10-16 $\mathrm{mm}$ and 16-25 mm stages. Generally, GhmiR157 abundance increased during floral organ development (Fig. 6a). In opened flowers, GhmiR157 was predominantly expressed in the anther and ovule, indicating that GhmiR157 may play a role in anther and ovule maturation. High levels of GhmiR157 could also be detected in the root, hypocotyl and leaf of seedlings (Fig. 6b).

The expression patterns of eight differentially expressed miR157-targeted GhSPLs (Fig. 5 and Additional file 6) were analysed during floral organ development. And statistically significant differences of expression level among floral organ development stages was analysed, based on analysis of variance. Since GhmiR157 could trigger degradation of its targeted mRNAs, the expression pattern of most miR157-targeted GhSPLs showed a decreasing trend during floral organ development. Gh_A01G0447, Gh_A01G2095, Gh_A11G0344, and Gh_A13G0749 were obviously down-regulated during the floral bud developmental stages (Fig. 6d, e, h and l). The abundance of Gh_A10G2217 and Gh_A11G2811 also slightly decreased (Fig. 6c and f). However, expression patterns of Gh_A04G1331 and Gh_A01G1281 did not show an obvious trend during floral bud development (Fig. $6 \mathrm{~g}$ and $\mathrm{m}$ ). According to negative correlation of expression pattern between GhmiR157 and most miR157-targeted GhSPLs, it is reasonable to speculate that GhmiR157 could trigger degradation of its targeted mRNAs to suppress the expression of miR157-targeted GhSPLs during floral organ development.

\section{Auxin signalling was attenuated in the over-expression lines}

Auxin plays an important role in flower development and floral organ size [21, 51, 52]. Intriguingly, some auxin-inducible genes, such as IAA-amido synthase, Auxin efflux carrier family protein and xyloglucan endotransglucosylase, were substantially down-regulated in two over-expression lines compared to Control (Fig. 7a-c). Other well-known auxin-inducible genes, IAA and SAUR, also had slightly lower expression in OV38 lines than the Control lines (Fig. 7d and e). To further verify the auxin signal difference between Control and over-expression lines, we used the plants transfected with the DR5::GUS vector to cross OV38 and Control plants, respectively. Since the DR5 promoter consists of seven repeats of an auxin-response element [53], the DR5::GUS reporter could 


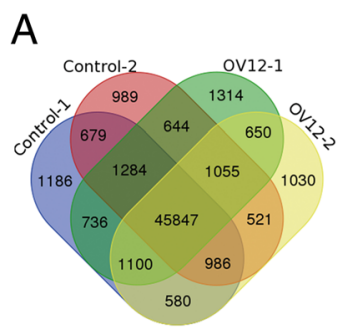

B
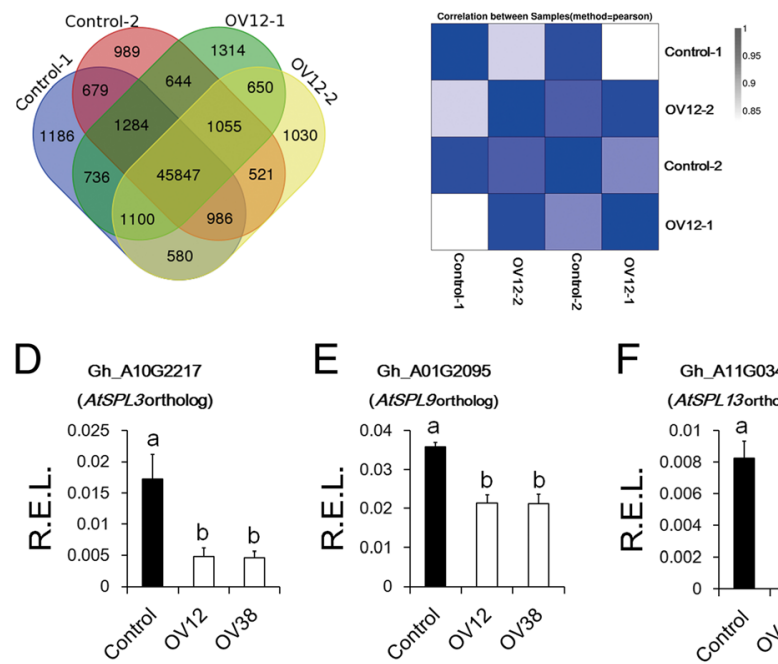

E

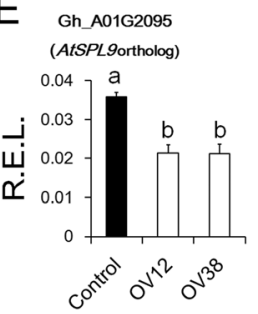

$\mathrm{F}$

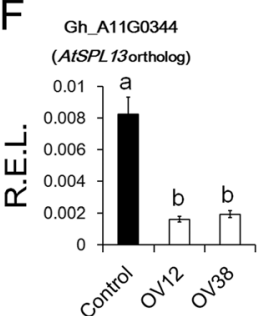

I

$\mathrm{H} \quad \begin{gathered}\text { Gh_A01G1281 } \\ \text { (AtSPL2ortholog) }\end{gathered}$

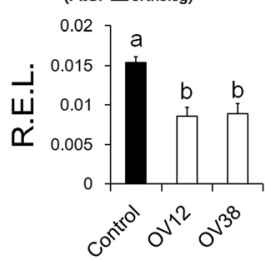
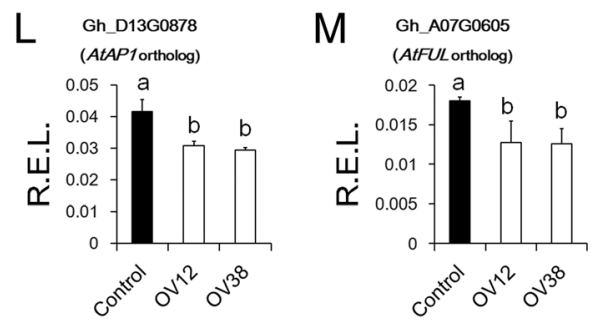

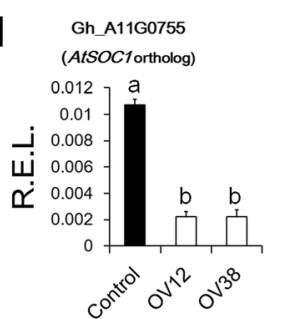

J
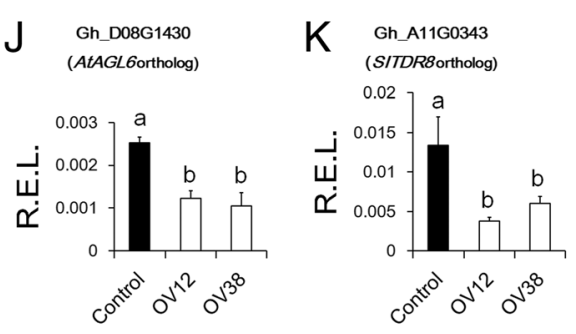

C

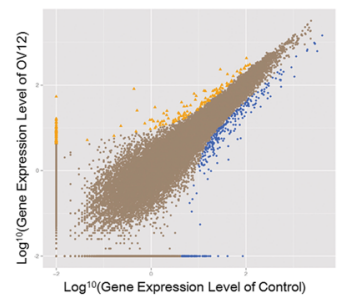

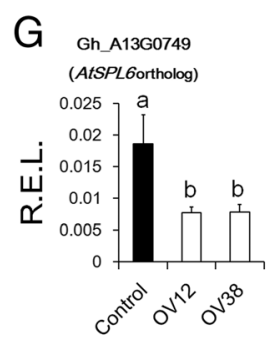

$\mathrm{N}$

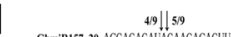

GimmiR157 20 ACGAGAGAUIAGGAGACAGLII

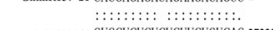$$
\text { A13 } 27323564 \text { GUGCUCLCUCUCUUCUGCLCAG } 2732354
$$
-

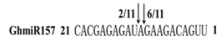

Fig. 5 RNA sequencing analysis of differentially expressed genes between Control and 35S::GhmiR157 transgenic line. a Venn diagram of co-expressed genes among samples. $\mathbf{b}$ Analysis of the correlation between samples. c Detection of differentially expressed genes between Control and 35S::GhmiR157 transgenic line based on the NOISeq method. Yellow plots indicate up-regulated genes in Control, blue plots indicate down-regulated genes in Control, brown plots indicate no changed genes. $\mathbf{d}$-m Real-time PCR analysis of differentially expressed genes in floral buds. R.E.L., the relative expression levels calculated using HISTONE3 (AF024716) as a control. The error bars indicate the standard deviation of four biological replicates. Different letters in the plots indicate statistically significant differences at $P<0.05$ based on analysis of variance (ANOVA) (Tukey's multiple comparison test). $\mathbf{n}$ GhmiR157 targets were identified using RLM-RACE. Black arrows indicate the position of the target cleavage sites. The numbers next to the black arrows indicate the cleavage frequency. Control, nontransgenic plant segregated from 35S::GhmiR157 transgenic lines in cotton. OV12 and OV38, 35S::GhmiR157 transgenic lines in cotton

be used to monitor the auxin response level. GUS signalling in floral buds and ovules was lower in DR5::GUS $\times$ OV38 than DR5::GUS $\times$ Control, indicating that auxin signalling was attenuated in the over-expression lines (Fig. $7 \mathrm{~g}$ and $\mathrm{h}$ ). However, transcript abundance of the key auxin biogenesis gene flavin monooxygenase (YUCCAs) did not show significant differences between Control and OV38 as determined by the RNA-sequencing results (Additional file 6). Additionally, free IAA content was conversely lower in Control than in OV12 and OV38 (Fig. 7f), which may indicate the existence of feedback regulation because the down-regulated AtGH3.6 ortholog in over-expression lines may decrease free IAA conjugation with amino acids. Thus, we concluded that auxin signal transduction was attenuated in over-expression lines.

\section{Discussion}

Recently, a lot of miRNAs have been identified through small RNA sequencing in cotton [43-46]. However, few of their function have been verified in cotton. In this 


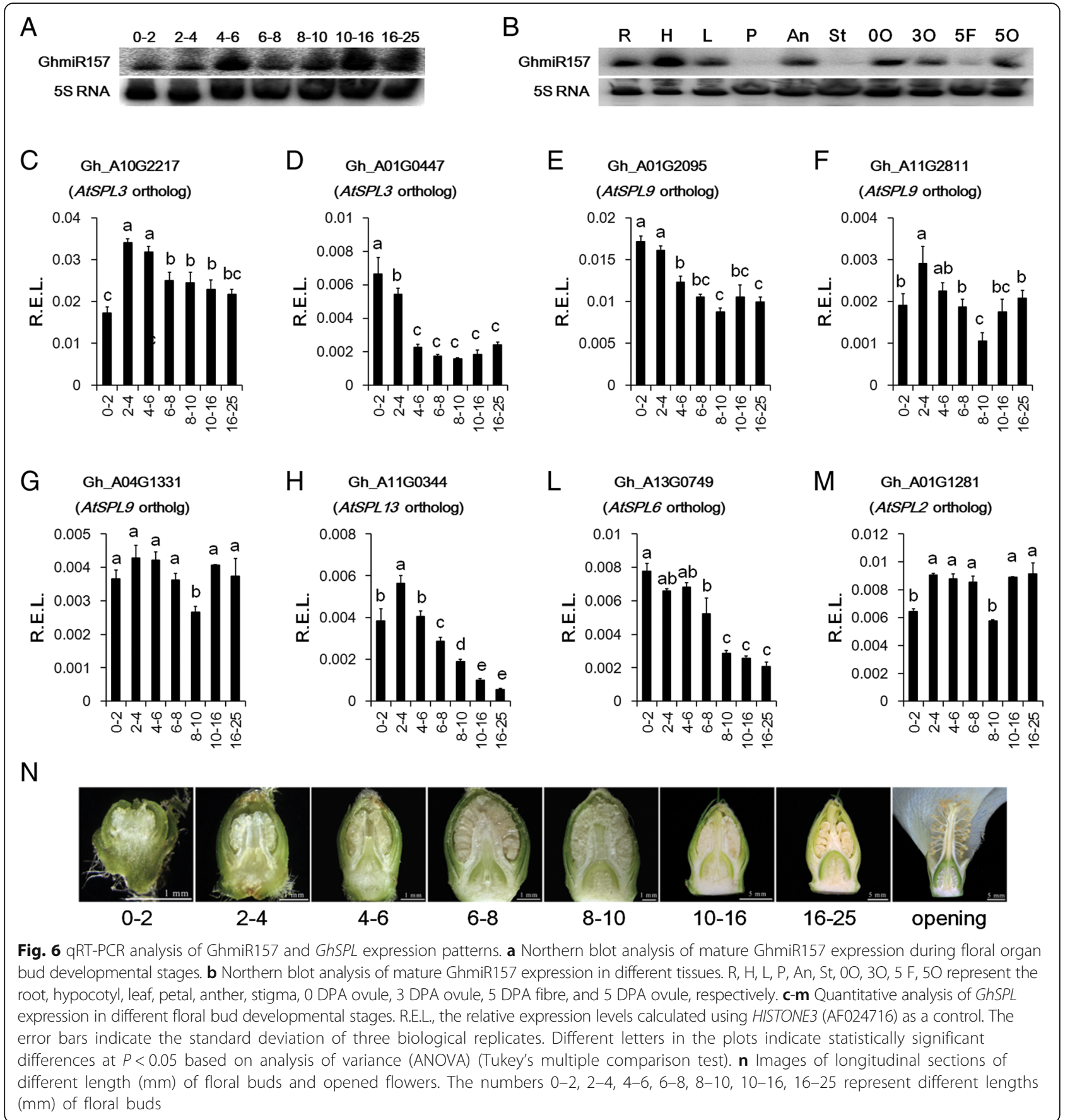

study, we found that over-expression of GhmiR157 in cotton could arrest cell proliferation and cell expansion, which repressed floral organ development and reduced the final organ size (Figs. 1 and 2, Table 1 and Additional file 4). Since floral bud growth was arrested at very early emergence, ovule primordium establishment may be repressed in the over-expression line, which resulted in reduced ovule production. All these results demonstrated that GhmiR157 may play an important role in floral organ development, although the mechanism needs to be further elucidated. Ectopic expression of the GhmiR157 precursor in Arabidopsis also reduced the petal area, gynoecium length and ovule number, which was similar to the phenotype in cotton. Since short gynoecium and few ovules were also found in 35S:AtMIR156b transgenic plants in Arabidopsis [54], it appears that the regulatory function in floral organ size and growth is conserved between species for miR156/ 157. Over-expressing the AtMIR156b precursor in tomato did not reduce floral organ size but resulted in 

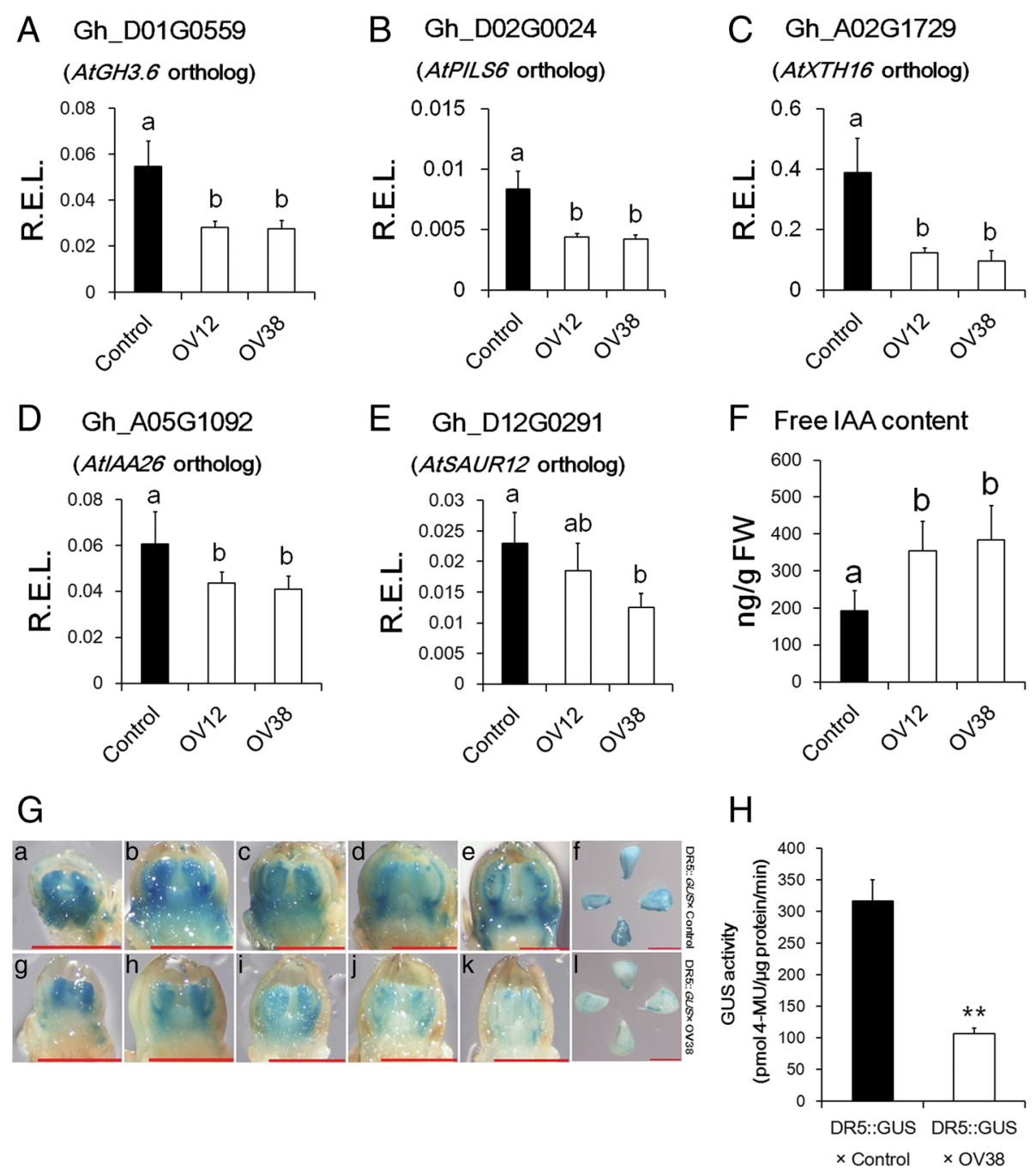

Fig. 7 Auxin signalling was lower in over-expression lines than Control. a-e Real-time PCR analysis of auxin-inducible genes in floral buds. R.E.L., the relative expression levels calculated using HISTONE3 (AF024716) as a control. The error bars indicate the standard deviation of four biological replicates. Different letters indicate statistically significant differences at $P<0.05$ based on analysis of variance (ANOVA) (Tukey's multiple comparison test). $\mathbf{f}$ Quantitative analysis of IAA content in the floral buds of Control, OV12 and OV38 at -1 DPA. The error bars indicate the standard deviation of at least six biological replicates. Different letters indicate statistically significant differences at $P<0.05$ based on analysis of variance (ANOVA) (Tukey's multiple comparison test). $\mathbf{g}$ GUS staining images of floral buds and ovules. Images of floral buds (length $\leq 2 \mathrm{~mm}$ ) from DR5::GUS $\times$ Control (a-e) and DR5::GUS $\times$ OV38 (g-k) and ovules from DR5::GUS $\times$ Control (f) and DR5::GUS $\times$ OV38 (I) at -1 DPA. Scale bar, 1 mm. h Quantitative GUS assays of ovules at -1 DPA. Asterisks indicate statistically significant differences at $P<0.01$ based on Student's $t$ test. Control, nontransgenic plant segregated from 35S::GhmiR157 transgenic lines in cotton. OV12 and OV38, 35S::GhmiR157 transgenic lines in cotton. DR5:GUS $\times$ Control and DR5::GUS $\times$ OV38 were F1 plants of DR5::GUS crossed with Control and OV38, respectively

severe fruit development defects, indicating that the miR156/157 family plays a major role in reproductive organ development among different species [55].

SPL transcription factors have been reported as miR156/ 157 targets in many species [42], and degradome sequencing also demonstrated that GhSPLs are targets of miR156/ 157 in cotton $[43,56]$. Additionally, in all the differentially expressed genes between the over-expression line and Control, only SPL transcription factors were predicted as miR157 targets, and five GhSPLs were further verified as miR157 targets using RLM-RACE (Fig. 5n and Additional file 7). The expression patterns of GhmiR157 and several targeted GhSPLs were generally negatively correlated during the floral organ developmental stages (Fig. 6). Therefore, it is reasonable to presume that GhSPLs as miR157 targets play important roles in floral organ development.

In Arabidopsis, AtSPLs could directly promote MADSbox transcription factors, such as AtSOC1, AtAP1, and AtFUL, to control phase transition [30-32]. In our study, the AtSOC1 ortholog and two other MADS-box transcription factors, the AtAGL6 ortholog and SITDR8 ortholog, were significantly down-regulated in the over-expression 
lines compared to Control (Fig. 5i-k and Additional file 6). The AGL6 clade of MADS-box genes is very similar to the closely related E class MADS-box genes and could serve as a scaffold to interact with other A, B, C and D class MADS-box transcription factors to form combinatorial quaternary complexes [57-59]. Dominant loss of function of the AGL6 clade of MADS-box genes by fusing a conserved suppressing motif to the proteins resulted in much smaller floral organs and partial sterility in Arabidopsis [58,60]. SITDR8 is another clade of MADS-box genes, which was not found in Arabidopsis [61]. Dominant loss of function of SITDR8 leads to alteration of ovary shape and seedless fruits [62]. MADS-box transcription factors play a crucial role in floral organ differentiation and development, and over-expressing miR157 in cotton resulted in smaller floral organs, fewer ovules and seeds, and attenuated female fertility, which partially reproduced the phenotype of mutants of these two genes in Arabidopsis and tomato. Therefore, it is likely that the miR157/SPL axis regulated orthologs of AtAGL6 and SITDR8 in cotton to control normal floral organ growth. However, direct regulation of these two clades of MADS-box genes in cotton by miR157-targeted GhSPLs should be verified in the future.

In addition to MADS-box transcription factors, several auxin-inducible genes were down-regulated in overexpression lines compared with Control (Fig. 7a-e). Moreover, auxin signalling, which could be monitored by the DR5::GUS reporter, was also lower in the overexpression lines than Control (Fig. $7 \mathrm{~g}$ and $\mathrm{h}$ ). However, free IAA content was not lower (Fig. 7f). Therefore, decreased auxin signalling in the over-expression lines was not due to IAA content but defects in signal transduction. The mechanism of how the miR157/SPL axis functions in auxin signal transduction is not clear. Interestingly, it was reported that the E class MADS-box transcription factor SEPALLATA3 could bind several ARF recognition motifs, for example, the AtGH3.3 promoter region [26]. Another study reported that MADS-box transcription factors could interact with ARF2 [29]. Given that AGL6 is functionally similar to SEPALLATA3, and the ortholog of AtAGL6 is down-regulated in over-expression lines, it is reasonable to hypothesize that MADS-box transcription factors, which may be regulated by miR157-targeted SPLs, possibly serve as ARF-like or ARF partner-like molecules to transduce auxin signalling to regulate normal organ differentiation and growth.
Finally, we propose a possible regulatory network of floral organ growth as described in Fig. 8. At floral bud emergence, there are low levels of GhmiR157 and little degradation of its targets, GhSPLs mRNAs. Highly expressed miR157-targeted GhSPLs may activate transcription of MADS-box genes, such as orthologs of AtAGL6 and SITDR8. These MADS-box transcription factors, or some unknown factors, may bind auxin response motifs of downstream gene promoters to transduce auxin signalling. Activated auxin signalling and MADS-box genes may further regulate downstream genes to establish normal organ primordium (such as ovules) and promote cell proliferation and cell expansion. In the late floral organ development stage, a high abundance of GhmiR157 decreases GhSPL expression to reduce the growth rate and accelerate floral organ (such as anther and ovule) maturation for fertilization.

\section{Conclusions}

Floral organs are crucial factors affecting the harvest of many crops and are complex reproductive organs that are regulated by many transcription factors and hormones [51]. Here, we found that the miR157/SPL axis could affect floral growth and size formation through regulating MADS-box genes and auxin signal transduction. The work further illuminates molecular basis of floral organ development, which is helpful for improvement of cotton yield. Future studies of the crosstalk between the miR157/ SPL axis and other factors in the regulation of floral organ development should be performed.

\section{Methods}

Plant materials and RNA isolation

Gossypium hirsutum cv. YZ1 was used as the wild type and transgenic receptor. All transgenic and nontransgenic cotton plants were grown in the experiment field and greenhouse at Huazhong Agricultural University in Wuhan using standard farming management practices in accordance with relevant national approvals for biotechnology research. The floral buds with lengths $\leq 2 \mathrm{~mm}$ and flower buds at minus one day post-anthesis ( -1 DPA) were harvested, immediately immersed in liquid nitrogen and stored at $-80{ }^{\circ} \mathrm{C}$. Total RNA was extracted using a thiocyanate method [63].

Arabidopsis thaliana ecotype Columbia was used as the wild type and transgenic receptor. Plants were grown in a greenhouse at $20^{\circ} \mathrm{C}-22{ }^{\circ} \mathrm{C}$ under long-day conditions

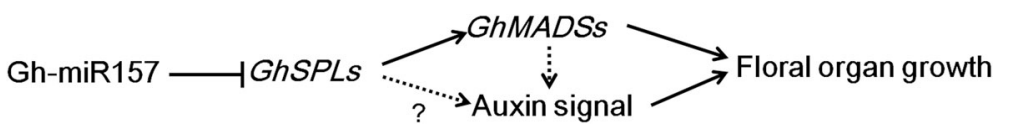

Fig. 8 A model for the potential miR157/SPLs axis in the regulatory network of floral organ development. The T shapes represent negative regulation. The dashed lines indicate hypothetical positive regulation 
(16 h light/8 h dark). Inflorescences were harvested, immersed in liquid nitrogen and stored at $-80{ }^{\circ} \mathrm{C}$. Total RNA was extracted using $\mathrm{TRIzol}^{\circledR}$ reagent according to the protocol (Thermo Fisher Scientific).

\section{Plasmid construction and genetic transformation}

A 372 bp genomic sequence containing a miR157 precursor from Gossypium hirsutum (Additional file 1) was cloned and ligated into the pGWB402 vector to overexpress the miR157 precursor [64]. A DR5 promoter fragment was ligated into the pGWB433 vector to construct the DR5::GUS vector [64]. The oligonucleotides for generating the plasmids described above are listed in Additional file 10.

Agrobacterium tumefaciens (GV3101) carrying the vector was used to transform hypocotyls of Gossypium hirsutum cv. YZ1. The infected hypocotyls were treated as described previously [65]. Agrobacterium tumefaciens (GV3101)-mediated transformation of Arabidopsis thaliana ecotype Columbia plants was performed by the floral dip method [66].

\section{Southern blotting, northern blotting and qRT-PCR analysis}

Southern blotting was performed as follows: genomic DNA isolation, enzyme digestion, electrophoresis and hybridization. The detailed methods were described previously [67]. The PCR-generated NPTII fragment was used as the probe. The relevant primers are listed in Additional file 10.

Northern blotting of miRNA was performed according to a previous report [43]. First, $20 \mu \mathrm{g}$ total RNA was electrophoresed in a $15 \%$ denaturing polyacrylamide gel containing $8 \mathrm{M}$ of urea and transferred to an Immobilon-Ny + membrane (Merck Millipore). Then, the probes were labelled with $\gamma 32 \mathrm{P}-\mathrm{ATP}$ using T4 polynucleotide kinase (New England BioLabs). After hybridization and membrane wash, the blot was exposed to a phosphor Imager screen, and the signal was detected in Cyclone Plus Phosphor Imager (PerkinElmer).

To quantify mRNA expression, $3 \mu \mathrm{g}$ total RNA was reverse-transcribed to cDNA using SuperScript II reverse transcriptase (Invitrogen). qRT-PCR was performed using a 7500 real-time system (Applied Biosystems) with SsoFast EvaGreen Supermix With Low ROX (Bio-Rad). The relative expression levels (R.E.L.) were calculated using the $2^{-\triangle C T}$ method. HISTONE3 (AF024716) and AtACT7 (AT5G09810.1) were used as endogenous reference genes in cotton and Arabidopsis respectively.

\section{Morphological and cellular analysis}

For kinematic analysis of cotton petal development, petals were manually dissected from the first-node floral bud on the second branches back to the first-node floral bud on the ninth branches when the first-node flower on the first branches opened.

For measurements of petal, sepal and bract area, the organs were flattened and scanned to produce a digital image. ImageJ software (https://imagej.nih.gov/ij/download.html) was used to calculate the organ areas. The cotton ovule, seed and anther number and stigma length were calculated manually. For measurement of ovule number in Arabidopsis, the gynoecium was washed in $70 \%$ ethanol twice and cleared in chloral hydrate: $\mathrm{dH}_{2} \mathrm{O}$ :glycerine (8:3:1). Ovules were observed using differential interference contrast microscopy (ZEISS).

Petal cell size was measured on the adaxial side at the top of petal. Average cell size was calculated from the number of cells per unit area of images from microscopy (ZEISS). Petal cell number was calculated according to cell size and petal area.

\section{Bioinformatic analysis of sequencing data}

Floral buds (length $\leq 2 \mathrm{~mm}$ ) were sampled from Control and miRNA157-over-expressing OV12 plants growing in a greenhouse. RNA libraries were generated and sequenced via Illumina $\mathrm{HiSeq}^{\mathrm{TM}} 2000$ at the Beijing Genomics Institute (BGI) in Shenzhen. After sequencing, the raw reads were filtered into clean reads and then mapped to the reference genome of Gossypium hirsutum [48] using Bowtie [68]. Gene expression levels were quantified using the software package RNASeq by Expectation Maximization [69]. Differentially expressed genes between the Control and OV12 lines with two biological repeats were screened using the NOISeq package [49].

miR157 target prediction was performed on a website tool using the default criterion [70].

\section{RNA ligase-mediated rapid amplification of CDNA end (RLM-RACE)}

RLM-RACE was performed with a GeneRacer kit (Invitrogen) to map the cleavage sites of target transcripts. Total RNA $(5 \mu \mathrm{g})$ from floral buds (length $\leq 2 \mathrm{~mm}$ ) was ligated to RNA adapters without calf intestine alkaline phosphatase. The cDNAs were transcribed using the GeneRacer Oligo $\mathrm{dT}$ primer. The PCR was performed with $5^{\prime}$ adaptor primers and 3 ' gene-specific primers according to the manufacturer's instructions. RACE products were cloned, and approximately 10 inserts were sequenced and analysed.

Histochemical analysis and quantification of GUS activity Floral buds (length $\leq 2 \mathrm{~mm}$ ) and ovules ( $-1 \mathrm{DPA})$ were incubated in the GUS staining buffer at $37{ }^{\circ} \mathrm{C}$ for $4 \mathrm{~h}$ and then washed in $75 \%$ ethanol one or more times. Stained samples were photographed using a stereomicroscope (Leica Microsystems). The staining buffer contained $0.9 \mathrm{~g} \mathrm{~L}^{-1}$ 5-bromo-4-chloro-3-indolylglucuronide, $50 \mathrm{mM}$ 
sodium phosphate buffer ( $\mathrm{pH} 7.0), 20 \%(\mathrm{v} / \mathrm{v})$ methanol and $100 \mathrm{mg} \mathrm{L}^{-1}$ chloromycetin.

For quantification of GUS activity, total protein from the samples was extracted using GUS extraction buffer containing $50 \mathrm{mM}$ potassium phosphate buffer at $\mathrm{pH} 7.0,10 \mathrm{mM}$ ethylenediaminetetraacetic acid, $0.1 \%$ sodium laurylsarcosine, $0.1 \%$ Triton $\mathrm{X}-100$ and $10 \mathrm{mM} \beta$ mercaptoethanol. The homogenate was centrifuged, and the supernatant was collected to measure GUS activity as described previously [71].

\section{Quantification of endogenous IAA}

Floral buds (100 mg fresh weight) at -1 DPA were homogenized in $1 \mathrm{~mL}$ of $80 \%$ (vol/vol) methanol containing $10 \mathrm{ng} /$ $\mathrm{mL}^{2} \mathrm{H}_{5}$-IAA (OIChemlm Ltd, CAS: 76937-78-5) as the internal standard and then shaken at $4{ }^{\circ} \mathrm{C}$ overnight. The supernatant was evaporated and redissolved in $10 \%$ (vol/ vol) methanol and subsequently filtered through a $0.22 \mu \mathrm{m}$ nylon membrane. The quantification of endogenous IAA was performed according to a previous report [72].

\section{Additional files}

Additional file 1: GhmiR157 precursor from Gossypium hirsutum. (A) Genomic sequence containing GhmiR157 precursor. The underline indicates mature miR157 sequence. (B) The secondary structure of GhmiR157 precursor. The red line indicates mature miR157 sequence. (DOCX $2223 \mathrm{~kb}$ )

Additional file 2: Positive test for 35S::GhmiR157 transformants. (A) Southern blot of 35S::GhmiR157 transformants. (B) PCR analysis of 35S::GhmiR157 transformants. Control, nontransgenic plant segregated from 35S::GhmiR157 transgenic lines in cotton. The numbers 11, 12, 33, $35,37,38,40$ represent different 35s::GhmiR 157 transgenic lines in cotton. (DOCX $287 \mathrm{~kb}$ )

Additional file 3: Over-expressing GhmiR157 precursor leads to more vegetative branches. (A-E) The pictures of plants at squaring stage in trial field from Control (A), OV12 (B), OV38 (C), OV33 (D), OV35 (E). (F and G) Plants at boll opening stage in field from Control $(\mathbf{F})$ and OV12 $(\mathbf{G})$. (H) Measurement of vegetative branches at squaring stage in green house. Control, nontransgenic plant segregated from 35S::GhmiR157 transgenic lines. OV12, 38, 33, 37 and 35, independent 35S::GhmiR157 transgenic lines. Different letters indicate statistically significant differences at $P<0.05$ based on analysis of variance (ANOVA) (Tukey's multiple comparison test). (DOCX $748 \mathrm{~kb}$ )

Additional file 4: Over-expressing GhmiR157 precursor suppressed reproductive organs development. (A) qRT-PCR of mature miR157 expression in floral buds. R.E.L., the relative expression levels calculated using HISTONE3 (AF024716) as a control. The error bars indicate the standard deviation of three biological replicates. Different letters indicate statistically significant differences at $P<0.05$ based on analysis of variance (ANOVA) (Tukey's multiple comparison test). (B-F) Images of flowers (B), stamens and stigmas (C), ovaries after removing the valves (D), 30 DPA bolls (E) and mature bolls (F). (G) The size of floral organs in WT and over-expressing GhmiR157 lines. Values are shown as the mean \pm standard deviation. In each column, values with different letters are significantly different based on Tukey's multiple comparison test $(P<$ 0.05). OV12, 38, 33 and 35, independent 35s::GhmiR157 transgenic lines. WT, wild type (Gossypium hirsutum cv. YZ1). Control, nontransgenic plant segregated from 35S::GhmiR157 transgenic lines. (DOCX $400 \mathrm{~kb}$ )

Additional file 5: Summary statistics of sequencing and mapping. (XLSX $10.1 \mathrm{~kb})$
Additional file 6: The abundance and annotation of genes in Control and OV12. (XLSX $8040 \mathrm{~kb}$ )

Additional file 7: Predicted targets of miR157. (XLSX $11.8 \mathrm{~kb}$ )

Additional file 8: Unrooted phylogram of all SPL genes in Gossypium hirsutum and Arabidopsis. Unrooted phylogram was performed by MEGA6 software based on the neighbor-joining algorithm. The red lines indicate the subfamily members are candidates of miR157 targets predicted through psRNATarget website tool [69]. (DOCX $48.2 \mathrm{~kb}$ )

Additional file 9: Quantification analysis of MADS-box genes in ectopicexpressing GhmiR157 precursor lines and wild type. (A-D) qRT-PCR of MADS-box genes in inflorescence. E4 and E6 indicate 35S::GhmiR157 transgenic lines in Arabidopsis. R.E.L., the relative expression levels calculated using AtACT7 (AT5G09810.1) as a control. The error bars indicate the standard deviation of four biological replicates. (DOCX $88.3 \mathrm{~kb}$ )

Additional file 10: Primer applied in the study. (XLSX $12.7 \mathrm{~kb})$

\section{Abbreviations}

AP1: APETALA1; FUL: FRUITFULL; SAUR: SMALL AUXIN UP RNA;

SOC1: SUPPRESSOR OF CONSTANS OVEREXPRESSION 1; SPL: SQUAMOSA

Promoter- Binding Protein- Like

\section{Acknowledgements}

We are grateful to Xu Jian (Department of Biological Science, National University of Singapore) for kindly providing the DR5 promoter and Nakagawa Tsuyoshi (Shimane University) for kindly providing the pGWB402 vector. We also thank Liu Hongbo and Li Dongqin (National Key Laboratory of Crop Genetic Improvement, Huazhong Agricultural University) for their assistance with the determination of IAA content.

\section{Funding}

This work was financially supported by the Program of Introducing Talents of Discipline to Universities in China (grant no. B14032) and the Fundamental Research Funds for the Central Universities (no. 2013YB06).

\section{Availability of data and materials}

RNA sequence data from floral buds (length $\leq 2 \mathrm{~mm}$ ) sampled from Control and miRNA157-over-expressing OV12 plants growing in a greenhouse has been submitted to The NCBI Sequence Read Archive (BioProject: PRJNA341749).

\section{Authors' contributions}

NL, LT and XZ designed the research; NL performed experiments and analyzed data; LW, HH and JX supply materials; NL wrote the manuscript and XZ revised the maunscript. All the Authors critically read and approved the final version of the manuscript.

\section{Competing interests}

The authors declare that they have no competing interests.

\section{Consent for publication}

Not applicable.

\section{Ethics approval and consent to participate}

Not applicable.

Received: 13 September 2016 Accepted: 23 December 2016 Published online: 10 January 2017

\section{References}

1. Hepworth J, Lenhard M. Regulation of plant lateral-organ growth by modulating cell number and size. Curr Opin Plant Biol. 2014;17:36-42.

2. Powell AE, Lenhard M. Control of organ size in plants. Curr Biol. 2012;22: R360-7.

3. Noir S, Bömer M, Takahashi N, Ishida T, Tsui TL, Balbi V, et al. Jasmonate controls leaf growth by repressing cell proliferation and the onset of endoreduplication while maintaining a potential stand-by mode. Plant Physiol. 2013;161(4):1930-51. 
4. Huang HY, Jiang WB, Hu YW, Wu P, Zhu JY, Liang WQ, et al. BR signal influences Arabidopsis ovule and seed number through regulating related genes expression by BZR1. Mol Plant. 2013;6(2):456-69.

5. Zhiponova MK, Vanhoutte I, Boudolf V, Betti C, Dhondt S, Coppens F, et al. Brassinosteroid production and signaling differentially control cell division and expansion in the leaf. New Phytol. 2013;197(2):490-502.

6. Lee BH, Ko JH, Lee S, Lee Y, Pak JH, Kim JH. The Arabidopsis GRFINTERACTING FACTOR gene family performs an overlapping function in determining organ size as well as multiple developmental properties. Plant Physiol. 2009;151(2):655-68.

7. Sauret-Güeto S, Schiessl K, Bangham A, Sablowski R, Coen E. JAGGED controls Arabidopsis petal growth and shape by interacting with a divergent polarity field. PLoS Biol. 2013;11(4):e1001550.

8. Anastasiou E, Kenz S, Gerstung M, MacLean D, Timmer J, Fleck C, et al. Control of plant organ size by KLUH/CYP78A5-dependent intercellular signaling. Dev Cell. 2007;13(6):843-56.

9. Xia T, Li N, Dumenil J, Li J, Kamenski A, Bevan MW, et al. The ubiquitin receptor DA1 interacts with the E3 ubiquitin ligase DA2 to regulate seed and organ size in Arabidopsis. Plant Cell. 2013;25(9):3347-59.

10. Li YH, Zheng LY, Corke F, Smith C, Bevan MW. Control of final seed and organ size by the DAl gene family in Arabidopsis thaliana. Genes Dev. 2008; 22(10):1331-6.

11. Xu R, Li Y. The mediator complex subunit 8 regulates organ size in Arabidopsis thaliana. Plant Signal Behav. 2012;7(2):182-3.

12. Ikeda M, Fujiwara S, Mitsuda N, Ohme-Takagi M. A triantagonistic basic helix-loop-helix system regulates cell elongation in Arabidopsis. Plant Cell. 2012;24(11):4483-97.

13. Bartrina I, Otto E, Strnad M, Werner T, Schmülling T. Cytokinin regulates the activity of reproductive meristems, flower organ size, cvule formation, and thus seed yield in Arabidopsis thaliana. Plant Cell. 2011;23(1):69-80.

14. Nelissen H, Rymen B, Jikumaru Y, Demuynck K, Van Lijsebettens M, Kamiya $Y$, et al. A local maximum in gibberellin levels regulates maize leaf growth by spatial control of cell division. Curr Biol. 2012;22(13):1183-7.

15. Hu Y, Xie Q, Chua NH. The Arabidopsis auxin-inducible gene ARGOS controls lateral organ size. Plant Cell. 2003;15(9):1951-61.

16. Krizek B. AINTEGUMENTA and AINTEGUMENTA-LIKE6 act redundantly to regulate Arabidopsis floral growth and patterning. Plant Physiol. 2009;150(4): $1916-29$.

17. Mizukami Y, Fischer RL. Plant organ size control: AINTEGUMENTA regulates growth and cell numbers during organogenesis. Proc Natl Acad Sci U S A. 2000;97(2):942-7.

18. Spartz AK, Lee SH, Wenger JP, Gonzalez N, Itoh H, Inzé D, et al. The SAUR19 subfamily of SMALL AUXIN UP RNA genes promote cell expansion. Plant J. 2012;70(6):978-90.

19. Varaud E, Brioudes F, Szécsi J, Leroux J, Brown S, Perrot-Rechenmann C, et al. AUXIN RESPONSE FACTOR8 regulates Arabidopsis petal growth by interacting with the bHLH transcription factor BIGPETALp. Plant Cell. 2011; 23(3):973-83.

20. Schruff MC, Spielman M, Tiwari S, Adams S, Fenby N, Scott RJ. The AUXIN RESPONSE FACTOR 2 gene of Arabidopsis links auxin signalling, cell division, and the size of seeds and other organs. Development. 2006;133:251-61.

21. Hawkins C, Liu Z. A model for an early role of auxin in Arabidopsis gynoecium morphogenesis. Front Plant Sci. 2014;5:327.

22. Galbiati F, Sinha Roy D, Simonini S, Cucinotta M, Ceccato L, Cuesta C, et al An integrative model of the control of ovule primordia formation. Plant J. 2013;76(3):446-55.

23. Theißen G. Development of floral organ identity: stories from the MADS house. Curr Opin Plant Biol. 2001;4(1):75-85

24. Theiszen G, Saedler H. Plant biology: floral quartets. Nature. 2001;409:469-71.

25. Dornelas MC, Patreze CM, Angenent GC, Immink RG. MADS: the missing link between identity and growth? Trends Plant Sci. 2011;16(2):89-97.

26. Kaufmann K, Muiño JM, Jauregui R, Airoldi CA, Smaczniak C, Krajewski P, et al. Target genes of the MADS transcription factor SEPALLATA3: integration of developmental and hormonal pathways in the Arabidopsis flower. PLoS Biol. 2009;7(4):e1000090.

27. Folter SD, Angenent GC. trans meets cis in MADS science. Trends Plant Sci. 2006;11(5):224-31

28. Ó'Maoiléidigh DS, Wuest SE, Rae L, Raganelli A, Ryan PT, Kwaśniewska K, et al. Control of reproductive floral organ identity specification in Arabidopsis by the C function regulator AGAMOUS. Plant Cell. 2013;25(7): 2482-503.
29. Smaczniak C, Immink RG, Muiño JM, Blanvillain R, Busscher M, BusscherLange J, et al. Characterization of MADS-domain transcription factor complexes in Arabidopsis flower development. Proc Natl Acad Sci U S A 2012:109(5):1560-5.

30. Yamaguchi A, Wu MF, Yang L, Wu G, Poethig RS, Wagner D. The microRNAregulated SBP-Box transcription factor SPL3 is a direct upstream activator of LEAFY, FRUITFULL, and APETALA1. Dev Cell. 2009;17(2):268-78.

31. Wang JW, Czech B, Weigel D. miR156-regulated SPL transcription factors define an endogenous flowering pathway in Arabidopsis thaliana. Cell. 2009; 138(4):738-49.

32. Xu M, Hu T, Zhao J, Park MY, Earley KW, Wu G, et al. Developmental functions of miR156-regulated SQUAMOSA PROMOTER BINDING PROTEIN-LIKE (SPL) genes in Arabidopsis thaliana. PLoS Genet. 2016;12(8):e1006263.

33. Si L, Chen J, Huang X, Gong H, Luo J, Hou Q, et al. OSSPL13 controls grain size in cultivated rice. Nat Genet. 2016:48:447-56.

34. Wang L, Sun S, Jin J, Fu D, Yang X, Weng $X$, et al. Coordinated regulation of vegetative and reproductive branching in rice. Proc Natl Acad Sci U S A. 2015;112:15504-9.

35. Wang S, Wu K, Yuan Q, Liu X, Liu Z, Lin X, et al. Control of grain size, shape and quality by OsSPL16 in rice. Nat Genet. 2012;44:950-4.

36. Jiao Y, Wang Y, Xue D, Wang J, Yan M, Liu G, et al. Regulation of OsSPL14 by OsmiR156 defines ideal plant architecture in rice. Nat Genet. 2010; 42(6):541-4.

37. Zhang TQ, Lian H, Tang H, Dolezal K, Zhou CM, Yu S, et al. An intrinsic microRNA timer regulates progressive decline in shoot regenerative capacity in plants. Plant Cell. 2015;27:349-60.

38. Yu N, Niu QW, Ng KH, Chua NH. The role of miR156/SPLs modules in Arabidopsis lateral root development. Plant J. 2015;83(4):673-85.

39. Stief A, Altmann S, Hoffmann K, Pant BD, Scheible WR, Bäurle I. Arabidopsis miR156 regulates tolerance to recurring environmental stress through SPL transcription factors. Plant Cell. 2014;26(4):1792-807.

40. Cui LG, Shan JX, Shi M, Gao JP, Lin HX. The miR156-SPL9-DFR pathway coordinates the relationship between development and abiotic stress tolerance in plants. Plant J. 2014:80:1108-17.

41. Chen X, Zhang Z, Liu D, Zhang K, Li A, Mao L. SQUAMOSA promoterbinding protein-like transcription factors: star players for plant growth and development. J Integr Plant Biol. 2010;52(11):946-51.

42. Wang $H$, Wang $H$. The miR156/SPL module, a regulatory hub and versatile toolbox, gears up crops for enhanced agronomic traits. Mol Plant. 2015;8(5): $677-88$

43. Liu N, Tu L, Tang W, Gao W, Lindsey K, Zhang X. Small RNA and degradome profiling reveals a role for miRNAs and their targets in the developing fibers of Gossypium barbadense. Plant J. 2014;80(2):331-44.

44. Xue W, Wang Z, Du M, Liu Y, Liu JY. Genome-wide analysis of small RNAs reveals eight fiber elongation-related and 257 novel microRNAs in elongating cotton fiber cells. BMC Genomics. 2013;14:629.

45. Gong L, Kakrana A, Arikit S, Meyers BC, Wendel JF. Composition and expression of conserved microRNA genes in diploid cotton (Gossypium) species. Genome Biol Evol. 2013:5:2449-59.

46. Pang M, Woodward AW, Agarwal V, Guan X, Ha M, Ramachandran V, et al. Genome-wide analysis reveals rapid and dynamic changes in miRNA and siRNA sequence and expression during ovule and fiber development in allotetraploid cotton (Gossypium hirsutum L.). Genome Biol. 2009:10:R122.

47. Cuperus JT, Fahlgren N, Carrington JC. Evolution and functional diversification of MIRNA genes. Plant Cell. 2011;23(2):431-42.

48. Zhang T, Hu Y, Jiang W, Fang L, Guan X, Chen J, et al. Sequencing of allotetraploid cotton (Gossypium hirsutum L. acc. TM-1) provides a resource for fiber improvement. Nat Biotechnol. 2015;33:531-7.

49. Tarazona S, García-Alcalde F, Dopazo J, Ferrer A, Conesa A. Differential expression in RNA-seq: a matter of depth. Genome Res. 2011;21(12):2213-23.

50. Leng N, Dawson JA, Thomson JA, Ruotti V, Rissman Al, Smits BMG, et al. EBSeq: an empirical Bayes hierarchical model for inference in RNA-seq experiments. Bioinformatics. 2013;29(8):1035-43.

51. Krizek BA, Anderson JT. Control of flower size. J Exp Bot. 2013;64(6):1427-37.

52. Sundberg E, Østergaard L. Distinct and dynamic auxin activities during reproductive development. Cold Spring Harb Perspect Biol. 2009;1 (6):575-83.

53. Xu J, Hofhuis H, Heidstra R, Sauer M, Friml J, Scheres B. A molecular framework for plant regeneration. Science. 2006;311:385-8.

54. Xing S, Salinas M, Garcia-Molina A, Hohmann S, Berndtgen R, Huijser P. SPL8 and miR156-targeted SPL genes redundantly regulate Arabidopsis gynoecium differential patterning. Plant J. 2013;75(4):566-77. 
55. Ferreira GF, Silva EM, Azevedo MS, Guivin MA, Ramiro DA, Figueiredo CR, et al. microRNA156-targeted SPL/SBP box transcription factors regulate tomato ovary and fruit development. Plant J. 2014;78(4):604-18.

56. Yang X, Wang L, Yuan D, Lindsey K, Zhang X. Small RNA and degradome sequencing reveal complex miRNA regulation during cotton somatic embryogenesis. J Exp Bot. 2013;64(6):1521-36.

57. Dreni L, Zhang D. Flower development: the evolutionary history and functions of the AGL6 subfamily MADS-box genes. J Exp Bot. 2016;67(6): 1625-38.

58. Hsu WH, Yeh TJ, Huang KY, Li JY, Chen HY, Yang CH. AGAMOUS-LIKE13, a putative ancestor for the $\mathrm{E}$ functional genes, specifies male and female gametophyte morphogenesis. Plant J. 2014;77(1):1-15.

59. Rijpkema AS, Zethof J, Gerats T, Vandenbussche M. The petunia AGL6 gene has a SEPALLATA-like function in floral patterning. Plant J. 2009;60(1):1-9.

60. Koo SC, Bracko O, Park MS, Schwab R, Chun HJ, Park KM, et al. Control of lateral organ development and flowering time by the Arabidopsis thaliana MADS-box Gene AGAMOUS-LIKE6. Plant J. 2010;62(5):807-16.

61. Smaczniak C, Immink RGH, Angenent GC, Kaufmann K. Developmental and evolutionary diversity of plant MADS-domain factors: insights from recent studies. Development. 2012;139(17):3081-98.

62. Margherita D, Simona M, Francesca R, Alessandro L, Giorgio C. Characterization of TM8, a MADS-box gene expressed in tomato flowers, BMC Plant Biol. 2014;14:319.

63. Zhu LF, Tu LL, Zeng FC, Liu DQ, Zhang XL. An improved simple protocol for isolation of high quality RNA from Gossypium spp. suitable for CDNA library construction. Acta Agron Sin. 2005:31:1657-9.

64. Nakagawa T, Suzuki T, Murata S, Nakamura S, Hino T, Maeo K, et al. Improved gateway binary vectors: high-performance vectors for creation of fusion constructs in transgenic analysis of plants. Biosci Biotechnol Biochem. 2007;71(8):2095-100

65. Jin S, Zhang X, Nie Y, Guo X, Liang S, Zhu H. Identification of a novel elite genotype for in vitro culture and genetic transformation of cotton. Biol Plant. 2006:50(4):519-24.

66. Zhang X, Henriques R, Lin SS, Niu QW, Chua NH. Agrobacterium-mediated transformation of Arabidopsis thaliana using the floral dip method. Nat Protoc. 2006; (2):641-6.

67. Li YJ, Liu DQ, Tu LL, Zhang XL, Wang L, Zhu LF, et al. Suppression of GhAGP4 gene expression repressed the initiation and elongation of cotton fiber. Plant Cell Rep. 2010;29(2):193-202.

68. Langmead B, Trapnell C, Pop M, Salzberg SL. Ultrafast and memory-efficient alignment of short DNA sequences to the human genome. Genome Biol. 2009;10(3):1-10.

69. Li B, Dewey CN. RSEM: accurate transcript quantification from RNA-Seq data with or without a reference genome. BMC Bioinformatics. 2011:12(1):93-9.

70. Dai X, Zhao PX. psRNATarget: a plant small RNA target analysis server. Nucleic Acids Res. 2011;39:W155-9.

71. Li Y, Tu L, Ye Z, Wang M, Gao W, Zhang X. A cotton fiber-preferential promoter, PGbEXPA2, is regulated by GA and ABA in Arabidopsis. Plant Cell Rep. 2015;34(9):1539-49.

72. Liu HB, Li XH, Xiao JH, Wang SP. A convenient method for simultaneous quantification of multiple phytohormones and metabolites: application in study of rice-bacterium interaction. Plant Methods. 2012;8:2.

\section{Submit your next manuscript to BioMed Central and we will help you at every step:}

- We accept pre-submission inquiries

- Our selector tool helps you to find the most relevant journal

- We provide round the clock customer support

- Convenient online submission

- Thorough peer review

- Inclusion in PubMed and all major indexing services

- Maximum visibility for your research

Submit your manuscript at www.biomedcentral.com/submit

) Biomed Central 